\title{
Real-Time Dynamics of the Formation of Hydrated Electrons upon Irradiation of Water Clusters with Extreme Ultraviolet Light
}

\author{
A. C. LaForge, ${ }^{1,2, *}$ R. Michiels, ${ }^{1}$ M. Bohlen, ${ }^{1}$ C. Callegari, ${ }^{3}$ A. Clark, ${ }^{4}$ A. von Conta, ${ }^{5}$ M. Coreno, ${ }^{6}$ M. Di Fraia, ${ }^{3}$ \\ M. Drabbels, ${ }^{4}$ M. Huppert, ${ }^{5}$ P. Finetti, ${ }^{3}$ J. Ma, ${ }^{5}$ M. Mudrich, ${ }^{7}$ V. Oliver, ${ }^{4}$ O. Plekan, ${ }^{3}$ K. C. Prince, ${ }^{3}$ M. Shcherbinin, ${ }^{7}$ \\ S. Stranges, ${ }^{8}$ V. Svoboda, ${ }^{5}$ H. J. Wörner, ${ }^{5}$ and F. Stienkemeier ${ }^{1,9}$ \\ ${ }^{1}$ Institute of Physics, University of Freiburg, 79104 Freiburg, Germany \\ ${ }^{2}$ Department of Physics, University of Connecticut, Storrs, Connecticut 06269, USA \\ ${ }^{3}$ Elettra-Sincrotrone Trieste, 34149 Basovizza, Trieste, Italy \\ ${ }^{4}$ Laboratory of Molecular Nanodynamics, Ecole Polytechnique Fédérale de Lausanne, 1015 Lausanne, Switzerland \\ ${ }^{5}$ Laboratorium für Physikalische Chemie, ETH Zürich, 8093 Zürich, Switzerland \\ ${ }^{6}$ ISM-CNR, Istituto di Struttura della Materia, LD2 Unit, 34149 Trieste, Italy \\ ${ }^{7}$ Department of Physics and Astronomy, Aarhus University, 8000 Aarhus C, Denmark \\ ${ }^{8}$ Department of Chemistry and Drug Technologies, University Sapienza, 00185 Rome, Italy, \\ and Tasc IOM-CNR, Basovizza, Trieste, Italy \\ ${ }^{9}$ Freiburg Institute of Advanced Studies (FRIAS), University of Freiburg, 79104 Freiburg, Germany
}

(Received 1 August 2018; published 4 April 2019)

\begin{abstract}
Free electrons in a polar liquid can form a bound state via interaction with the molecular environment. This so-called hydrated electron state in water is of fundamental importance, e.g., in cellular biology or radiation chemistry. Hydrated electrons are highly reactive radicals that can either directly interact with DNA or enzymes, or form highly excited hydrogen $\left(\mathrm{H}^{*}\right)$ after being captured by protons. Here, we investigate the formation of the hydrated electron in real-time employing extreme ultraviolet femtosecond pulses from a free electron laser, in this way observing the initial steps of the hydration process. Using timeresolved photoelectron spectroscopy we find formation timescales in the low picosecond range and resolve the prominent dynamics of forming excited hydrogen states.
\end{abstract}

DOI: 10.1103/PhysRevLett.122.133001

The hydration of electrons in aqueous solutions plays a ubiquitous role in biological and chemical systems [1-3] and is of interest in atmospheric chemistry such as aerosol nucleation [4]. However, a fundamental understanding of the hydration properties has yet to be attained. In particular, the formation times, vertical binding energies (VBE), and binding motifs of the hydrated state are still under debate [5-9]. The hydrated electron is in a weakly bound state which can be ionized by UV radiation leading to a low kinetic energy, free electron, which is known to cause DNA damage $[10,11]$. Furthermore, the hydrated electron is a highly reactive radical which undergoes various chemical reactions with its surroundings. Formation of excited hydrogen via proton-transfer from water or recombination with $\mathrm{H}^{+}$complicates the assessment of its biological significance for radiation damage [1]. To address these issues experimentally, a major focus has been to directly measure the binding energy of the hydrated electron via photoelectron spectroscopy, which is sensitive to the strength of the electron-water interaction. The reported vertical binding energies range from 0.5 to $2.5 \mathrm{eV}$ for small anionic clusters and from 3.5 to $4.0 \mathrm{eV}$ for electrons hydrated in large clusters or bulk material [1,12]. Various experimental techniques have been employed to create a hydrated state in the laboratory: for example, using (i) multiple IR-UV photons to excite pure water to hydrated or prehydrated states [13], (ii) solute-doped water to initiate hydration via charge transfer to the solvent (CTTS) $[14,15]$, or (iii) electron attachment to small size-selected clusters [16]. In particular, for anionic water clusters, a large number of experiments on the hydrated electron have been performed revealing a rich structure of binding motifs or isomers for small cluster sizes [16-19]. In addition, timeresolved measurements have been performed to study excitation [20], charge transfer [21,22], and prehydration $[13,23,24]$ dynamics in various water systems. So far, the methods to study electron hydration have suffered from complex schemes (e.g., charge transfer to solvent, multiphoton ionization, anionic clusters), which complicate the understanding of the underlying mechanism.

In contrast, here, we use femtosecond extreme ultraviolet (XUV) free electron laser (FEL) radiation to directly study the hydration process. The XUV pulse initially ionizes the water clusters resulting in the creation of low-kineticenergy electrons. Through elastic and inelastic scattering, some of the electrons are trapped within the cluster forming bound, hydrated states. With a second UV pulse, we probe the process in time recording the resulting electron kinetic 


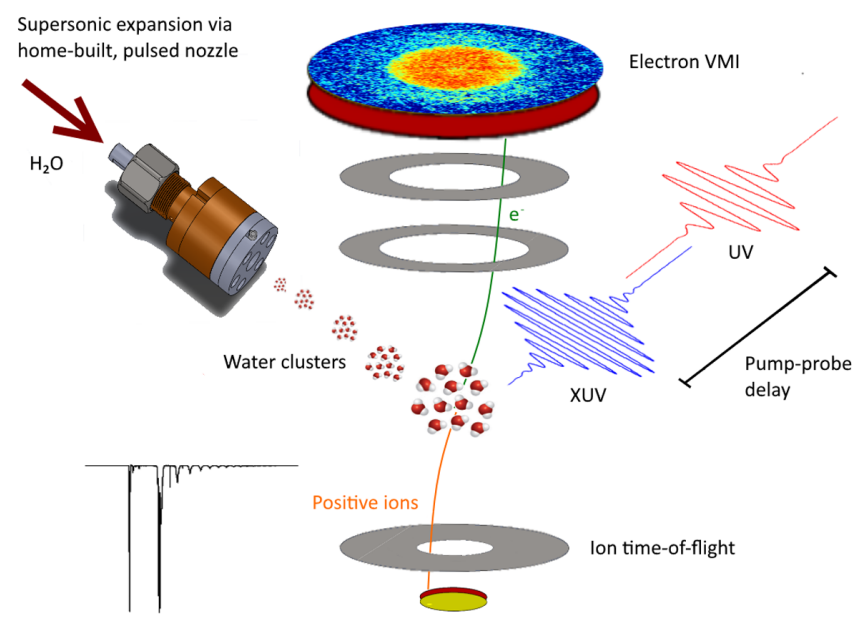

FIG. 1. Schematic of the experiment. The water clusters are created via supersonic expansion of heated liquid water through a home-built, pulsed nozzle. XUV radiation excites the clusters, creating quasifree electrons. The dynamics are measured by means of XUV pump-UV probe spectroscopy using photoelectron velocity-map imaging and photoion time-of-flight mass spectrometry.

energy distribution. The process is schematically shown in Fig. 1. From this, we map out the hydration process in time and determine the formation as well as decay times in the low picosecond range and simultaneously observe the formation of free, excited hydrogen atoms as a fast, dominant radiation product. Comparison with experiments on deuterated water $\left(\mathrm{D}_{2} \mathrm{O}\right)$, ammonia $\left(\mathrm{NH}_{3}\right)$, and methanol $(\mathrm{MeOH})$ clusters aids in the interpretation [25]. The experimental proof that a hydrated electron can be created directly via radiation is an important step in understanding most ionization-induced processes in aqueous solutions.

The experiment was performed at the Low Density Matter end station [29] at the seeded FEL, FERMI, in Trieste, Italy. The FEL photon energy, primarily set to $h \nu=24.0 \mathrm{eV}$, was tuned via the seed laser and by setting the undulator gaps, and other machine parameters, according to predefined values with a pulse length of approximately 100 fs FWHM [30,31]. The FEL pulse energy at the setup, approximately $70 \mu \mathrm{J}$ with a spot size of $20 \mu \mathrm{m}$ FWHM, is calculated from the value measured upstream on a shot-by-shot basis by gas ionization and the nominal reflectivity of the optical elements in the beam transport system. The UV pulse is produced from a frequency-tripled Ti:sapphire laser $(h \nu=4.75 \mathrm{eV})$ with a pulse energy of about $45 \mu \mathrm{J}$ and a spot size of $80 \mu \mathrm{m}$ FWHM. The temporal resolution between the two pulses was about 150 fs. A supersonic gas jet of water clusters is produced by expansion of heated liquid water through a home-built, pulsed nozzle. By varying the temperature of the liquid water sample, we can control the mean cluster size in the range of $10^{1}-10^{3}$ water molecules. For this experiment, a mean cluster size of 250 molecules was chosen to optimize signal intensity. The cluster beam was perpendicularly crossed by the FEL/UV beam at the center of a velocity map imaging spectrometer in combination with an ion time-of-flight mass spectrometer [29] schematically shown in Fig. 1. The kinetic energy distributions were reconstructed using the Maximum Entropy Legendre Reconstruction method [32].

As a first step, we address the UV $(h \nu=4.75 \mathrm{eV})$ ionized photoelectron spectrum, which was extracted by subtracting an XUV only $(h \nu=24.0 \mathrm{eV})$ spectrum from an $\mathrm{XUV}+\mathrm{UV}$ spectrum for each pump-probe delay step. The XUV + UV and XUV only spectra along with the subtraction method are given in the Supplemental Material [25]. To ensure the UV-ionized spectra were independent of the XUV FEL photon energy, the photon energy was tuned over a wide range $(h \nu=19.2-33.7 \mathrm{eV})$. Additionally, the FEL pulse energy was varied from approximately 2 to $70 \mu \mathrm{J}$ to confirm the power dependence was linear for both XUV-ionized and UV-ionized electrons [25]. The UVionized electrons also showed a linear power dependence with respect to the UV laser power. Plotted in Fig. 2 is the UV-ionized photoelectron spectrum as a function of the vertical binding energy. For all figures, vertical electronic transitions were assumed. The pump-probe delay was $20 \mathrm{ps}$, which corresponds to a time where all measured fast dynamics have subsided. The spectra were fitted with a multi-Gaussian function to better understand the distribution. The first striking feature in the spectrum is the pair of sharp peaks at $1.5 \pm 0.05$ and $3.5 \pm 0.05 \mathrm{eV}$ which have a full width at half maximum (FWHM) of $0.3 \pm 0.05 \mathrm{eV}$. The width of these contributions is mainly due to the instrumental broadening of the spectrometer. This clearly points towards atomic states that are not affected by the cluster environment and, indeed, the corresponding binding energies match excited states of atomic hydrogen which are marked in blue in Fig. 2. The distributions were additionally confirmed by comparison to other hydrogen-bonded clusters, $\mathrm{NH}_{3}$ and $\mathrm{MeOH}$ [25]. Additional atomic features were not observed at least within the experimental resolution. Furthermore, the positions and widths of these distributions do not change with respect to the molecule and experimental variables (FEL photon energy, FEL pulse energy, and cluster size). As such, these parameters were constrained in the Gaussian fit function allowing for a more precise fitting of additional, overlapping peaks in the spectra. The next prominent feature in Fig. 2 is the broad peak to the right of the $\mathrm{H}^{*}(n=2)$ photoelectrons, centered at $3.9 \pm 0.15 \mathrm{eV}$ with a FWHM of about $0.9 \pm 0.15 \mathrm{eV}$. By comparison with the well-known energies of hydrated electronic states [1], we assign this distribution to electrons hydrated inside the water clusters, labeled $e_{a q}^{-}$ in Fig. 2. Note that for $\mathrm{NH}_{3}$ and $\mathrm{MeOH}$ a distribution was not observed at this vertical binding energy, further confirming that it is due to the hydrated electron in water clusters [25]. In terms of the binding energy, there are slight 


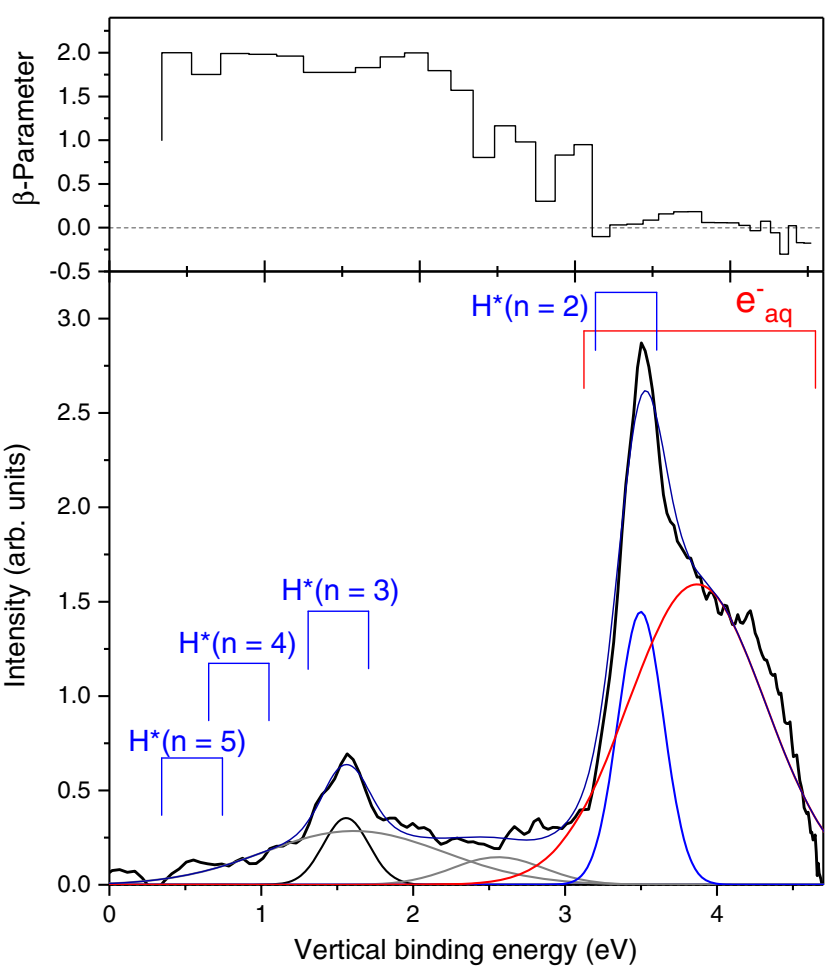

FIG. 2. Photoelectron intensities from water clusters are shown as a function of binding energy at a XUV-UV pump-probe delay of $20 \mathrm{ps}$ for $24.0 \mathrm{eV}$ FEL photon energy. This spectrum shows only the UV probe-ionized electrons which are obtained by subtracting spectra with XUV + UV and XUV only for each delay step. The contributions from excited hydrogen states and their expected binding energies are marked in blue and black whereas the hydrated electron VBE distributions are marked in red. For the contributions marked in gray, an unambiguous assignment was not possible. The anisotropy parameter $\beta$ is shown in the top panel.

discrepancies with respect to previous work on anionic clusters; however, taking into consideration the influence of the charge state, our results still fit well in terms of both the energy and width of the distribution. In general, the binding energies of the hydrated electron are dependent on the location, and the conventional understanding is that surface-bound hydrated electrons have a lower binding energy compared to internally bound hydrated states [18]. The broad gray peaks at 1.7 and $2.6 \mathrm{eV}$ could possibly fit to additional binding motifs such as the surface-bound hydrated electron. However, in this region, the assignment is more ambiguous due to the contributions of the higher excited hydrogen states; thus we do not quantify this further. As an important difference with respect to the previous results on anionic clusters [1], we cannot confirm a significant shift in VBE for the hydrated electron as a function of cluster size in the range of $\langle n\rangle=250$ to $\langle n\rangle=$ 2200 molecules [25]. This can possibly be explained by the method in which the hydrated electrons are created. For anionic clusters, electrons are first associated with neutral clusters, then experimentally size selected. On the other hand, for the current work, the formation of the hydrated state is determined by the capability of the water cluster to initially capture the electron, which is dependent on the geometrical size of the cluster and, thereby, favorable to larger clusters.

The top panel of Fig. 2 shows the anisotropy parameter $\beta$, which represents the angular distribution of the emitted electrons [33]. For lower binding energies ( $\mathrm{VBE} \leq 2.5 \mathrm{eV})$, in the vicinity of the higher-lying excited atomic hydrogen states $\left[\mathrm{H}^{*}(n \geq 3)\right]$, the distributions are highly anisotropic with a $\beta$ parameter close to 2 . This indicates the distributions originate from atomic states detached from the water cluster. On the other hand, in the vicinity of the hydrated electron peak, the $\beta$ parameter is nearly isotropic $(\beta \approx 0.2)$, similar to previous studies on electron hydration in liquid water [34]. This gives further evidence of our observation of a hydrated electron. More than likely, the anisotropy of the $\mathrm{H}^{*}(n=2)$ state is washed out by the isotropy of the hydrated electron peak. This interpretation is corroborated by the results of other hydrogen-bonded clusters $\left(\mathrm{NH}_{3}\right.$ and $\left.\mathrm{MeOH}\right)$ where all $\mathrm{H}^{*}$ states show a anistropic distribution of $\beta \approx 2$ [25].

To better understand how FEL radiation can efficiently hydrate electrons, one can turn to recent theoretical results on intense, $\mathrm{x}$-ray absorption in hydrogen-bonded clusters for a viable explanation [35]. When atomic clusters are irradiated with intense, energetic light, the clusters become highly ionized leading to Coulomb explosion. On the other hand, the presence of hydrogen leads to completely different effects where the free electrons migrate to the bulk of the cluster and the protons are efficiently ejected resulting in the cluster remaining intact and in a low charge state. The collective result of these effects is ideal for creating a suitable environment for electron hydration. Additionally, it helps to explain the large presence of excited hydrogen that we observe.

Let us now turn to the dynamics of the individual processes. In Fig. 3, plotted as a function of the pumpprobe delay are the evolution of the VBE in the top panel and the electron yields from the area of the Gaussian fits from Fig. 2 in the lower panel. The hydrated electron forms with an exponential rise time of $\tau_{1}=(1.3 \pm 0.5) \mathrm{ps}$ and has a partial decay afterwards with a time constant $\tau_{2}=(4.5 \pm 0.6) \mathrm{ps}$. Since the hydrated electron is expected to have a lifetime that depends on the availability of partners for secondary processes, we attribute this decay to the recombination with excess protons created via photodissociation of water molecules. This assumption is backed by the biexponential rise time of the excited hydrogen peaks involving two different time constants. The first rise is $\tau_{1}=(0.2 \pm 0.2) \mathrm{ps}$, which is significantly faster than the formation of the hydrated electron and is only slightly above the temporal resolution of our experiment $(\approx 150 \mathrm{fs})$. This corresponds to excited hydrogen atoms which are created from immediate recombination 


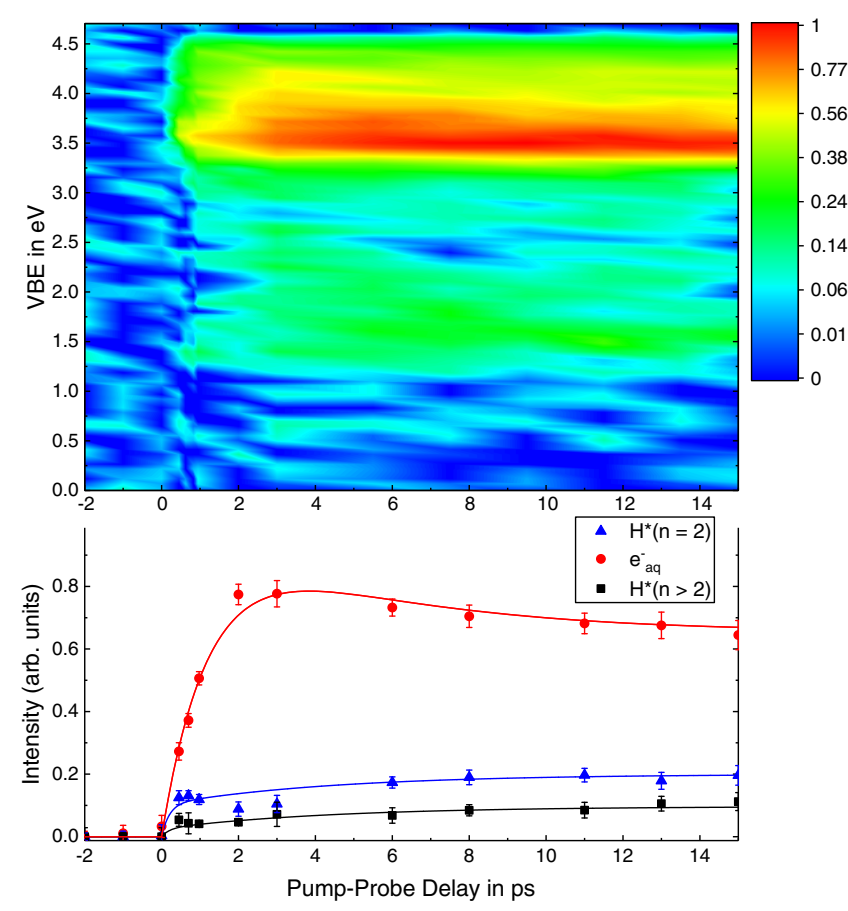

FIG. 3. Top panel: Color plot of the VBE as a function of the pump-probe time delay. The $z$ axis is the square root of the intensity with no baseline subtraction. Bottom panel: electron yields from the area of the Gaussian fits from Fig. 2 as a function of the pump-probe delay. The hydrated electron is fitted with a fast exponential rise of $\tau_{1}=1.3 \mathrm{ps}$ and a slow exponential decay with $\tau_{2}=4.5 \mathrm{ps}$. The excited hydrogen intensity is fitted with a biexponential rise with $\tau_{1}=0.2$ and $\tau_{2}=4.5 \mathrm{ps}$. The spectra were taken with a FEL photon energy of $24.0 \mathrm{eV}$. The error bars represent the statistical uncertainties in the Gaussian fitting procedure between pump-probe delay steps.

of thermalized electrons with ejected protons created by the XUV pulse. The second time constant is $\tau_{2}=(4.5 \pm 0.6) \mathrm{ps}$, which is compatible with the decay constant of the hydrated electron and therefore agrees with the interpretation of these excited hydrogen atoms being created via recombination of hydrated electrons with protons inside the cluster. After formation and initial recombination, the remaining free electrons enter a longlived bound state, the hydrated state. The formation dynamics of the excited hydrogen additionally gives information on how such a state is formed. It is known that the formation of excited hydrogen is a major decay channel of the hydrated electron in the vicinity of free protons [1].

Our time-resolved results clearly show that the formation of excited hydrogen can be a dominant initial process when irradiating water clusters with intense XUV light. In general, there are three ways to create excited hydrogen atoms with XUV radiation. The first option is dissociative photoexcitation of $\mathrm{H}_{2} \mathrm{O}$ into $\mathrm{OH}$ and $\mathrm{H}^{*}(n=2)$ which has been reported for molecular water [36], but this is only a minor contribution in our experiment due to the low cross section [37]. A second option is the direct recombination of quasi-free electrons with $\mathrm{H}^{+}$after photodissociation of $\mathrm{H}_{2} \mathrm{O}$ into $\mathrm{OH}^{-}$and $H^{+}$. A third option would be the recombination of a hydrated electron with excess $\mathrm{H}^{+}$ cations created by (dissociative) multiple ionization events taking place in the same water cluster. One can distinguish between the second and the third option through their time dependence, since the formation of a fully hydrated state requires more time because it involves molecular rearrangement. We find formation times of hundreds of femtoseconds (fs) for the second process and a picosecond (ps) timescale for the third process, which is delayed by the electron hydration with a time constant of $1.3 \mathrm{ps}$. These findings correspond well to previous studies using transient-absorption spectroscopy $[13,38]$.

In conclusion, we have observed, in real-time, the electron hydration process using the XUV pump-UV probe technique. We determined a formation time of $\tau_{1}=$ $(1.3 \pm 0.5) \mathrm{ps}$ and see a recombination with free protons on a timescale of $\tau_{2}=(4.5 \pm 0.6) \mathrm{ps}$. This is the first time that the direct formation of the hydrated electron after single-photon ionization of water has been monitored. In particular, the results suggest that the proton concentration has a significant impact on the lifetime and reaction products of the hydrated electron. The possibility to create hydrated electrons with an XUV laser opens new perspectives for a number of important, future experiments. Additionally, we identify the formation of excited hydrogen produced via electron-proton recombination as a dominant, fast channel. The abundant formation of excited hydrogen also occurs in ammonia and methanol and appears to be universal.

Funding from the Deutsche Forschungsgemeinschaft (STI 125/19-1, GRK 2079), Carl-Zeiss-Stiftung, ETH Zürich, Grants No. 200021_146598 and No. 200020_162434 from the Swiss National Science Foundation, as well as the Department of Excellence, Department of Chemistry and Technologies of drugs, and Progetto Ateneo-2016 (Prot. No. RG116154C8E02882) of Sapienza University are gratefully acknowledged.

*aaron.laforge@uconn.edu

[1] M. P. Coons and J. M. Herbert, Annu. Rev. Phys. Chem. 68, 447 (2017).

[2] G. V. Buxton, C. L. Greenstock, W. P. Helman, and A. B. Roos, J. Phys. Chem. Ref. Data 17, 513 (1988).

[3] B. C. Garrett et al., Chem. Rev. 105, 355 (2005).

[4] F. Arnold, Nature (London) 294, 732 (1981).

[5] D. Luckhaus, Y.-i. Yamamoto, T. Suzuki, and R. Signorell, Sci. Adv. 3, e1603224 (2017).

[6] R. E. Larsen, W. J. Glover, and B. J. Schwartz, Science 329, 65 (2010).

[7] L. Turi and Á. Madarász, Science 331, 1387c (2011). 
[8] L. D. Jacobson and J.M. Herbert, Science 331, 1387d (2011).

[9] R. E. Larsen, W. J. Glover, and B. J. Schwartz, Science 331, 1387e (2011).

[10] B. Boudaiffa, P. Cloutier, D. Hunting, M. A. Huels, and L. Sanche, Science 287, 1658 (2000).

[11] E. Alizadeh and L. Sanche, Chem. Rev. 112, 5578 (2012).

[12] K. Majer, Ph.D. thesis, Albert-Ludwigs-Universiät Freiburg im Breisgau, 2016.

[13] P. Kambhampati, D. H. Son, T. W. Kee, and P. F. Barbara, J. Phys. Chem. A 106, 2374 (2002).

[14] M. H. Elkins, H. L. Williams, A. T. Shreve, and D. M. Neumark, Science 342, 1496 (2013).

[15] J. P. Müller, N. Zhavoronkov, I. V. Hertel, and C. P. Schulz, J. Phys. Chem. A 118, 8517 (2014).

[16] J. Kima, I. Becker, O. Cheshnovsky, and M. A. Johnson, Chem. Phys. Lett. 297, 90 (1998).

[17] J. R. R. Verlet, A. E. Bragg, A. Kammrath, O. Cheshnovsky, and D. M. Neumark, Science 307, 93 (2005).

[18] J. V. Coe, S. M. Williams, and K. H. Bowen, Int. Rev. Phys. Chem. 27, 27 (2008).

[19] L. Ma, K. Majer, F. Chirot, and B. von Issendorff, J. Chem. Phys. 131, 144303 (2009).

[20] A. E. Bragg, J. R. R. Verlet, A. Kammrath, O. Cheshnovsky, and D. M. Neumark, Science 306, 669 (2004).

[21] J. Stähler, J.-C. Deinert, D. Wegkamp, S. Hagen, and M. Wolf, J. Am. Chem. Soc. 137, 3520 (2015).

[22] A. Kothe, M. Wilke, A. Moguilevski, N. Engel, B. Winter, I. Y. Kiyan, and E. F. Aziz, Phys. Chem. Chem. Phys. 17, 1918 (2015).

[23] A. Migus, Y. Gauduel, J. L. Martin, and A. Antonetti, Phys. Rev. Lett. 58, 1559 (1987).

[24] J. C. Alfano, P. Walhout, Y. Kimura, and P. F. Barbara, J. Chem. Phys. 98, 5996 (1993).

[25] See Supplemental Material at http://link.aps.org/supplemental/ 10.1103/PhysRevLett.122.133001 for additional experimental details, and for information on pump-power and wavelength dependence as well as comparison with methanol and ammonia, which includes Refs. [26-28].

[26] G. N. Haddad and J. A. R. Samson, J. Chem. Phys. 84, 6623 (1986).

[27] Y. Itikawa and N. Mason, J. Phys. Chem. Ref. Data 34, 1 (2005).

[28] T. Fennel, K.-H. Meiwes-Broer, J. Tiggesbäumker, P.-G. Reinhard, P. M. Dinh, and E. Suraud, Rev. Mod. Phys. 82, 1793 (2010).

[29] V. Lyamayev, Y. Ovcharenko, R. Katzy, M. Devetta, L. Bruder, A. LaForge, M. Mudrich, U. Person, F. Stienkemeier, M. Krikunova et al., J. Phys. B 46, 164007 (2013).

[30] E. Allaria, R. Appio, L. Badano, W. Barletta, S. Bassanese, S. Biedron, A. Borga, E. Busetto, D. Castronovo, P. Cinquegrana et al., Nat. Photonics 6, 699 (2012).

[31] E. Allaria, A. Battistoni, F. Bencivenga, R. Borghes, C. Callegari, F. Capotondi, D. Castronovo, P. Cinquegrana, D. Cocco, M. Coreno et al., New J. Phys. 14, 113009 (2012).

[32] B. Dick, Phys. Chem. Chem. Phys. 16, 570 (2014).

[33] D. Parker and A. Eppink, J. Chem. Phys. 107, 2357 (1997).

[34] Y.-i. Yamamoto, Y.-I. Suzuki, G. Tomasello, T. Horio, S. Karashima, R. Mitríc, and T. Suzuki, Phys. Rev. Lett. 112, 187603 (2014).

[35] P. Di Cintio, U. Saalmann, and J.-M. Rost, Phys. Rev. Lett. 111, 123401 (2013).

[36] A. Hans, A. Knie, P. Schmidt, L. Ben Ltaief, C. Ozga, P. Reiß, H. Huckfeldt, M. Förstel, U. Hergenhahn, and A. Ehresmann, Phys. Rev. A 92, 032511 (2015).

[37] J. E. Mentall, G. R. Möhlmann, and P. M. Guyon, J. Chem. Phys. 69, 3735 (1978).

[38] V. H. Vilchiz, J. A. Kloepfer, A. C. Germaine, V. A. Lenchenkov, and S. E. Bradforth, J. Phys. Chem. A 105, 1711 (2001). 